\title{
WHAT IS THE EMITTANCE OF THE INJECTED BEAM?
}

\author{
G. Parzen
}

January 10, 1988 
The usual statement is that the remittance of the injected beam is $\varepsilon_{x}=\varepsilon_{y}=10$ (normalized) for heavy ions in RHIC.

Tracking studies require a more precise statement. Tracking requires that a. 4-dimenlonal surface be specified in $x, x^{\prime}, y, y^{\prime}$ space that contains $95 \%$ of the beam. The tracking studies can then investigate the stability of the particles in side this surface.

a simple way to specify this surface is by

$$
\varepsilon_{T}=\varepsilon_{x}\left(x, x^{\prime}\right)+\varepsilon_{y}\left(y, y^{\prime}\right)=C \text {, }
$$

where $C$ is a constant chosen So that this surface contains $95 \%$ of the beam. We reason for using this expression is that $\varepsilon_{T}$ is aery roughly a consent of the motion. 
2.

It will be argued below that the proper choice of $C$ is

$$
C=10(5 / 3)=16.7
$$

corresponding to the statement that $\varepsilon_{x}=\varepsilon_{y}=10$.

Assuming for the moment that $C=16.7$ is the proper choice of $C$, then in tracking studies where runs are done with $\varepsilon_{x}=\varepsilon_{y}$ then the proper starting remittance is

$$
\varepsilon_{x}=\varepsilon_{x}=8.33
$$

If runs are done with $\varepsilon_{y}=0$, then - the starting $\varepsilon_{x}$ is

$$
\begin{aligned}
& \varepsilon_{x}=16.7 \\
& \varepsilon_{y}=0
\end{aligned}
$$

These taro points are on the surface $\varepsilon_{T}=$ constant that contains 95\% of the beam. 
3

Iassume that the statement $\varepsilon_{x}=\varepsilon_{y}=10$ means that for the projection of the particles on the $x, x^{\prime}$ plane, 95\%. of the particles have an $\varepsilon_{x}$ which is smaller than $\varepsilon_{x}=10$, and a similar statement apply to the $y_{\text {, }} y^{\prime}$ plane.

I assume that the distribution $\rho\left(x, x^{\prime}, y, y^{\prime}\right)$ is gaussian with the form

$$
\rho\left(x, x^{\prime}, y, y^{\prime}\right) \sim \exp \left(-\left(\varepsilon_{x}\left(x, x^{\prime}\right)+\varepsilon_{y}\left(y_{y} y^{\prime}\right)\right) / \hat{\varepsilon}\right.
$$

The projection on the $x x^{\prime}$ plane has the distribution

$$
\begin{aligned}
\rho\left(x, x^{\prime}\right) & =\int d y d y^{\prime} \rho\left(x, x^{\prime}, y, y^{\prime}\right) \\
& \sim \exp \left(-\varepsilon_{x}\left(x, x^{\prime}\right) / \bar{\varepsilon}\right)
\end{aligned}
$$

In order for $95 \%$ of the particle to have an $\varepsilon_{x}$ which is smaller then $\varepsilon_{x}=10$ then

$$
\bar{\varepsilon}=10 / 3
$$


4

The fraction of the particles that have a total emitlance, $\varepsilon_{T}=\varepsilon_{x}+\varepsilon_{y}$, which is smaller than $\varepsilon_{T}$ is given by

$$
F\left(\varepsilon_{T}\right)=1-\exp \left(-\varepsilon_{T} / \bar{\varepsilon}\right)\left(1+\varepsilon_{T} / \bar{\varepsilon}\right)
$$

This may be derived from $E_{q}(1)$ for $\rho\left(x, x^{\prime}, y, y^{\prime}\right)$. The choice $\varepsilon_{T}$ that includes $95 \%$ of the particles is $\quad \varepsilon_{T}=5 \bar{\varepsilon}$ (the actual answer is closer to $4.8 \bar{\varepsilon})$.

Thu o the $\varepsilon_{T}$ that contains 95\% of the particles is

$$
\begin{aligned}
& \varepsilon_{T}=5 \bar{\varepsilon} \\
& \varepsilon_{T}=5\left(\frac{10}{3}\right) \\
& \varepsilon_{T}=16.67
\end{aligned}
$$


5

I wish to thank Herald Hahn for his suggestion for the meaning $o f$ the statement that $\varepsilon_{x}=10$. 